Ann. Génét. Sél. anim., I974, 6 (2), 275-28I.

NOTE

\title{
LA RACE PORCINE VIETNAMIENNE $I$ ' ET SON CROISEMENT AVEC LA RACE BERKSHIRE
}

\author{
TRAN THE THONG (1) \\ Institut national de Recherches zootechniques, \\ Hanoi, Nord-Vietnam
}

\section{RÉSUMÉ}

La race I' est une race de petite taille remarquable par sa précocité sexuelle surtout chez les mâles et par sa prolificité. La taille de la portée au sevrage est cependant limitée par le faible nombre de tétines chez la truie. Le croissment avec la race Berkshire permet d'améliorer la croissance et la composition corporelle des produits : gain de $22 \mathrm{~kg}$ à ro mois et augmentation du pourcentage de viande de 9 points par rapport à la race pure. Les truies de croisement $\left(I^{\prime} \times\right.$ Berkshire $)$ ont une prolificité diminuée, mais le poids moyen du porcelet à la naissance est accru de $70 \mathrm{p}$. Ioo par rapport à la race pure. Le nombre de tétines est intermédiaire entre les deux races parentales.

L'objet de cette note est de présenter les caractéristiques zootechniques de la principale race porcine de la plaine du Nord-Vietnam, la race $I^{\prime}$, et de son croisement avec la race Berkshire. Les données ont été recueillies dans des fermes expérimentales et des coopératives agricoles entre I962 et r 969 .

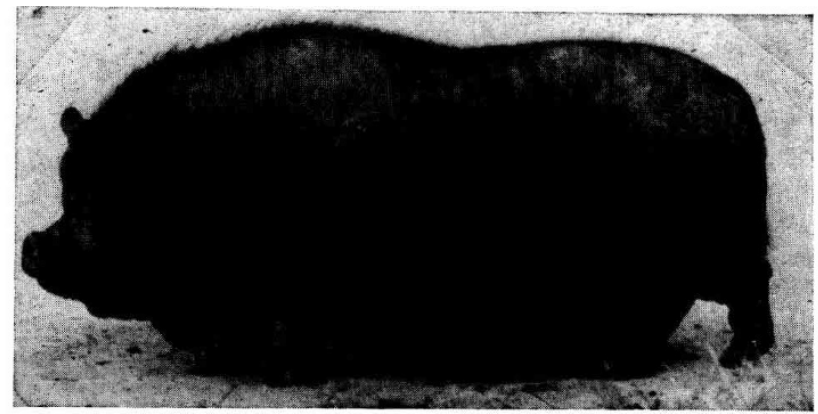

FIG. I. - Truie de race I'

(1) Tirés-à-part disponibles au Département de Génétique animale, C. N. R. Z., 78350 Jouy en Josas (France). 
Le Porc $I^{\prime}$ est de petite taille et présente un groin retroussé, un front étroit et plissé, un dos ensellé et un ventre très développé, traînant à terre chez les truies en lactation (fig. I). Sa robe est noire uniforme ainsi que la peau. C'est une race adaptée aux conditions tropicales du SudEst asiatique et à une nourriture à base principalement de plantes aquatiques très riches en eau et peu nutritives.

\section{I. - CARACTÈRES DE REPRODUCTION}

La race est remarquable par sa précocité sexuelle. Chez la femelle les premières chaleurs apparaissent à partir de $\mathbf{r} 20$ jours, elles durent de 3 à 8 jours et la durée du cycle varie de $\mathbf{I} 7$ à 23 jours. Le mâle peut saillir à partir de 45 jours. A go jours, le verrat donne de 60 à $70 \mathrm{~cm}^{3} \mathrm{de}$ semence par éjaculat avec une concentration de ro à 120 millions de spermatozoïdes par $\mathrm{cm}^{3}$. Le verrat adulte (à partir de 200 jours) donne un volume de 100 à $120 \mathrm{~cm}^{3}$ mais avec une concentration plus faible de $\mathrm{I} 2$ à $40 \times \mathrm{ro}^{6}$ spermatozoïdes par $\mathrm{cm}^{3}$. Dans la pratique, les verrats sont utilisés à partir d'environ 5 mois (soit environ $15 \mathrm{~kg}$ ).

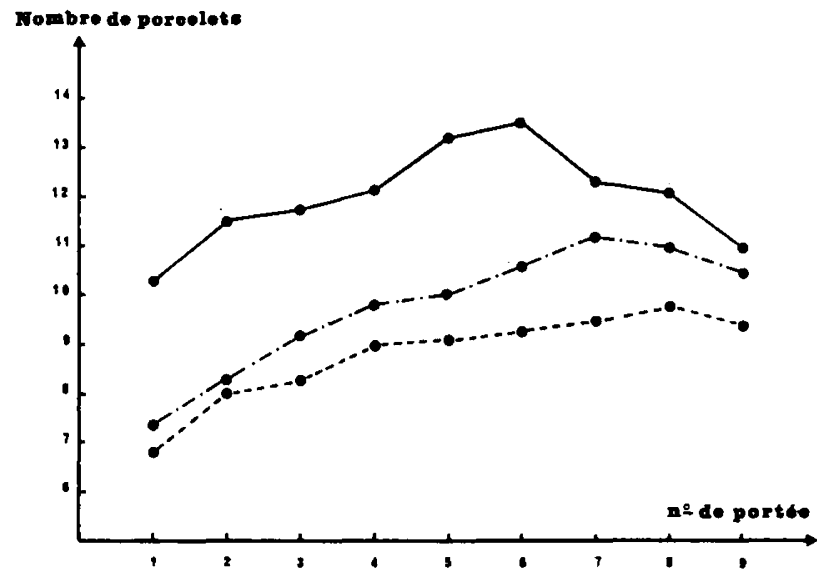

FIG. 2. - Evolution de la taille moyenne des portées en fonction du numéro de portée

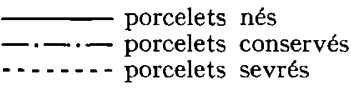

TABLEAU I

Influence de l'âge à la 1re saillie

\begin{tabular}{c|c|c|c|c}
\hline $\begin{array}{c}\text { Nombre } \\
\text { de truies }\end{array}$ & $\begin{array}{c}\text { Age à la } \\
\text { 1re saillie } \\
\text { (mois) }\end{array}$ & $\begin{array}{c}\text { Poids avant } \\
\text { la saillie } \\
(\mathrm{kg})\end{array}$ & $\begin{array}{c}\text { Nombre de } \\
\text { porcelets nés }\end{array}$ & $\begin{array}{c}\text { Nombre } \\
\text { de porcelets } \\
\text { conservés }\end{array}$ \\
\hline & & - & 9,2 & 7,0 \\
15 & 6 & 18,5 & 10,5 & 8,5 \\
15 & 7 & 29,0 & 11,0 & 9,0 \\
$11 k$ & 8 & 40,0 & 11,3 & 9,2 \\
14 & 9 & 43,0 & 11,2 & 9,1 \\
12 & 10 & 47,0 & & \\
\hline \hline
\end{tabular}


La prolificité des truies varie en fonction du numéro de portée comme indiqué dans la figure 2. Un maximum de plus de $\mathrm{I} 3$ porcelets nés vivants semble atteint vers la $5^{\mathrm{e}}$ portée. Malheureusement le nombre de tétines est insuffisant et oblige l'éleveur à une élimination importante de porcelets dès la naissance. Le tableau I montre comment varient le poids de la truie et la prolificité en fonction de l'âge à la première saillie fécondante. On voit que l'effet de l'âge est surtout important avant 8 mois.

\section{II. - CROISSANCE PONDÉRALE}

Le tableau 2 montre comment évolue le poids du porcelet de la naissance au sevrage dans les conditions courantes de l'élevage vietnamien (coopératives). La croissance jusqu'à ro mois, dans les conditions des fermes expérimentales, est donnée dans la figure 4 . Le poids des truies augmente jusqu'à la deuxième portée et se ralentit ensuite (tabl. 3). Les pertes de poids sont de l'ordre de Io p. Ioo après la mise bas et de 20 p. I כ en fì d'allaitement par rapport au poids avant la mise bas.

TABLEAU 2

Poids moyen des porcelets de 0 à 60 jours $(\mathrm{kg})$

\begin{tabular}{|c|c|c|c|}
\hline & Naissance & 30 jours & 60 jours \\
\hline Effectif ..... & 5560 & 4330 & 3890 \\
\hline Moyenne ..... & 0,425 & 2,396 & 4,695 \\
\hline Écart-type ........ & 0,120 & 0,480 & 1,170 \\
\hline
\end{tabular}

TABLEAU 3

Évolution pondérale des truies en reproduction

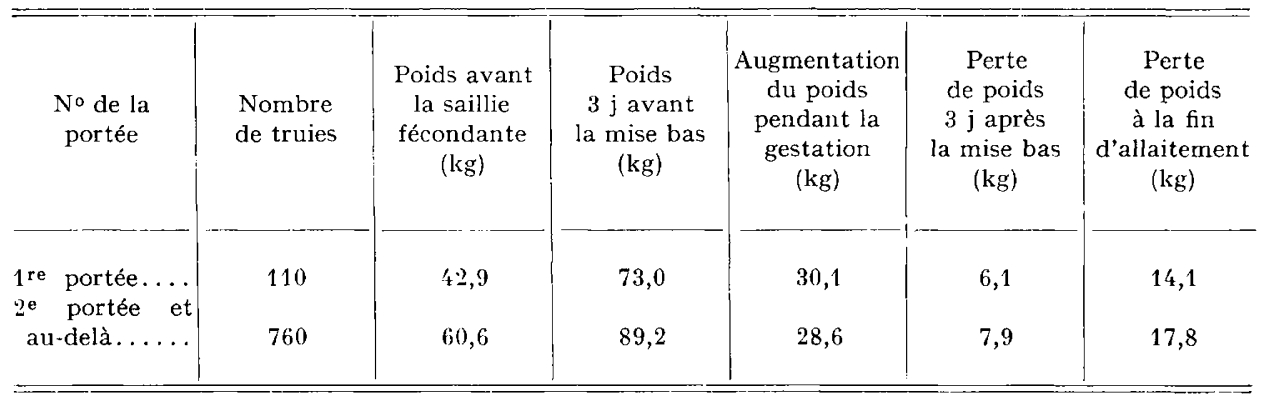

\section{III. - LE CROISEMENT' I' $\times$ BERKSHIRE}

Des croisements ont été effectués dans les porcheries expérimentales de l'I. N. K. Z., avec des verrats Berkshire, Large White et Landrace en provenance d'U. R. S. S. et de Chine. Nous nous limiterons aux résultats obtenus avec la première de ces trois races (fig. 3). 


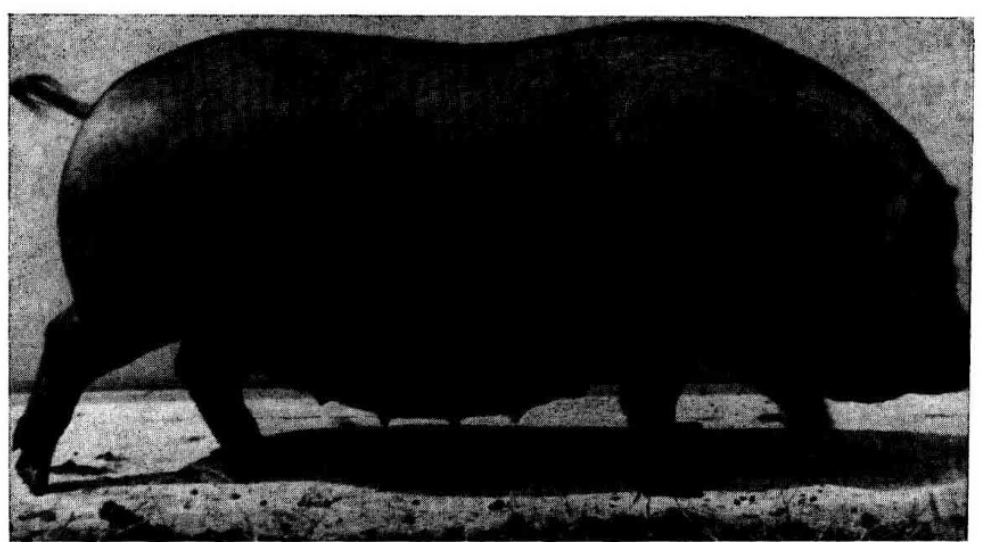

FIg. 3. - Femelle issue du croisement o Berkshire $\times$ \& I'

Le tableau 4 résume les effets de ce croisement sur la prolificité, le poids à la naissance des porcelets et le nombre de tétines. On voit que le mâle Berkshire a peu d'effet sur la prolificité des truies $I^{\prime}$ mais qu'il augmente notablement le poids de leur portée à la naissance. La femelle de croisement a une prolificité réduite mais elle donne des porcelets dont le poids à la naissance est augmenté de $70 \mathrm{p}$. Ioo par rapport à la race pure. Quant au nombre de tétines, il est très voisin, chez la femelle de croisement, de la moyenne des races parentales.

\section{TABLEAU 4}

Résultat du croisement I' $\times$ Berkshire sur les caractères de reproduction

\begin{tabular}{|c|c|c|c|c|c|c|c|}
\hline \multirow[b]{3}{*}{ No de portée } & \multicolumn{4}{|c|}{ q $I^{\prime}$} & \multirow{3}{*}{ 우 $B K$} & \multirow{2}{*}{\multicolumn{2}{|c|}{$\begin{array}{c}q \mathrm{~F}_{1}\left({ }^{1}\right) \\
\left(\sigma^{*} B K \times q I^{\prime}\right)\end{array}$}} \\
\hline & \multicolumn{2}{|c|}{ en race pure } & \multicolumn{2}{|c|}{ croisée avec ơ $B K$} & & & \\
\hline & 1 & 2 & 1 & 2 & & 1 & 2 \\
\hline $\begin{array}{l}\text { Nombre de portées } \ldots \ldots \ldots \\
\text { Nombre de porcelets con- } \\
\text { servés/portée } \ldots \ldots \ldots \ldots \\
\text { Poids moyen à la naissance } \\
\quad(\mathrm{kg}) \ldots \ldots \ldots \ldots \ldots \ldots \ldots\end{array}$ & $\begin{array}{c}30 \\
8,5 \\
0,450\end{array}$ & $\begin{array}{c}155 \\
11,4 \\
0,470\end{array}$ & $\begin{array}{c}25 \\
8,8 \\
0,580\end{array}$ & $\begin{array}{r}137 \\
12,0 \\
0,600\end{array}$ & $\begin{array}{l}- \\
- \\
-\end{array}$ & $\begin{array}{c}32 \\
8,0 \\
0,770\end{array}$ & $\begin{array}{c}112 \\
10,3 \\
0,800\end{array}$ \\
\hline Tétines & & & & & & & \\
\hline $\begin{array}{l}\text { Nombre d'animaux } \ldots \ldots \\
\text { Nombre moyen de tétines } \\
\pm \sigma \ldots \ldots \ldots \ldots \ldots \ldots \ldots \\
\text { Pourcentage ayant un nbre } \\
\text { pair de tétines } \ldots \ldots \ldots \\
\text { Pourcentage ayant plus de } \\
12 \text { tétines } \ldots \ldots \ldots \ldots \ldots \ldots\end{array}$ & & $\begin{array}{r}10,69 \\
2\end{array}$ & 0,91 & & $\begin{array}{c}240 \\
13,31 \pm 1,15 \\
67,18 \\
95,80\end{array}$ & $12,00=$ & $\begin{array}{l}18 \\
1,25 \\
25\end{array}$ \\
\hline
\end{tabular}

(1) En croisement avec $\delta I^{\prime}$. 
La croissance du porc de croisement, relativement à celle du porc $I^{\prime}$, est représentée dans la figure 4 . On voit qu'à Io mois la différence de poids est de $2 \mathrm{I}, 7 \mathrm{~kg}$. De plus l'indice de consommation du porc croisé entre le sevrage et l'abattage est amélioré de o,55 UF. Le tableau 5 montre l'amélioration qu'apporte également ce croisement du point de vue de la composition corporelle.

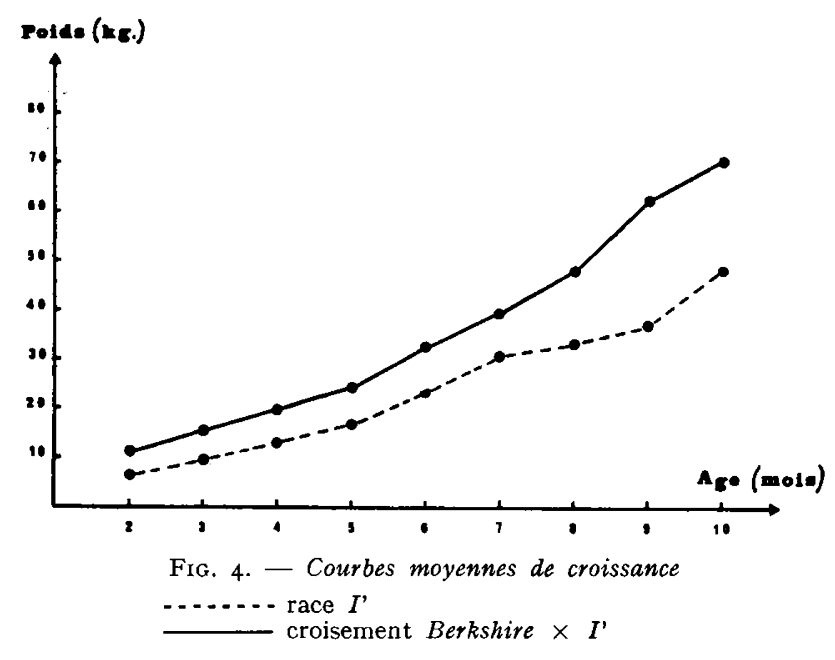

TABLEAU 5

Composition corporelle de porcs I' et $F_{1}\left(\mathrm{BK} \times \mathrm{I}^{\prime}\right)$

\begin{tabular}{|c|c|c|c|c|c|c|c|}
\hline \multirow[b]{2}{*}{ Race } & \multicolumn{7}{|c|}{ Critères } \\
\hline & $n$ & $\begin{array}{c}\text { Poids avant } \\
\text { l'abattage } \\
(\mathrm{kg})\end{array}$ & $\begin{array}{c}\text { P. } 100 \\
\text { carcasse } \\
\text { (avec tête) } \\
\text { sur poids vif }\end{array}$ & $\begin{array}{c}\text { P. } 100 \\
\text { carcasse } \\
\text { (sans tête) } \\
\text { sur poids vif }\end{array}$ & $\begin{array}{l}\text { P. } 100 \\
\text { graisse } \\
\text { dans la } \\
\text { carcasse }\end{array}$ & $\begin{array}{l}\text { P. } 100 \\
\text { viande } \\
\text { dans la } \\
\text { carcasse }\end{array}$ & $\begin{array}{l}\text { P. } 100 \text { os } \\
\text { dans la } \\
\text { carcasse }\end{array}$ \\
\hline I' & 12 & 47,0 & 73,0 & 67,1 & 41,9 & 34,5 & 10,6 \\
\hline$F_{1}$ & 12 & 76,0 & 81,0 & 67,9 & 34,8 & 43,9 & 8,9 \\
\hline
\end{tabular}

Ces résultats expliquent la vogue que connaît actuellement ce croisement au Vietnam.

\section{III. - CONCLUSION}

Le tableau 6 récapitule les principales caractéristiques de la race vietnamienne $I^{\prime}$ (comparativement à celles du Large White européen). Il s'agit d'une race bien adaptée aux climats chauds, très précoce, prolifique et rustique. Dans un premier stade, le croisement, facilité par l'insémination artificielle, a pu apporter une amélioration notable de la production de viande. Par ailleurs, un programme de sélection de la race pure est entrepris, qui vise à améliorer la croissance, la composition corporelle et le nombre de tétines pour permettre une meilleure exploitation de la prolificité naturelle de la race. Ce programme d'amélioration génétique, basé sur l'utilisation du croise- 
ment industriel entre des verrats occidentaux et des truies asiatiques améliorées, devrait donner assez rapidement de bons résultats, la structure de l'élevage porcin au Nord-Vietnam étant favorable à la mise en place de tels programmes.

\section{TABLEAU 6}

Comparaison de la race I' au Large White européen

\begin{tabular}{|c|c|c|c|c|c|}
\hline Reproduction & $I^{\prime}$ & $L . I V$. & $\begin{array}{c}\text { Croissance et } \\
\text { développement }\end{array}$ & $I^{\prime}$ & L. $W$. \\
\hline 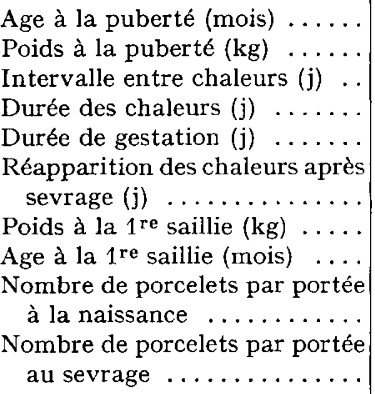 & $\begin{array}{c}3-4 \\
12-15 \\
21 \\
3-8 \\
110-112 \\
24 \\
30-40 \\
8-10 \\
11,8 \\
8,3\end{array}$ & $\begin{array}{c}6-8 \\
110 \\
21 \\
2-3 \\
115 \\
\\
12-15 \\
100-120 \\
6-8 \\
10,8 \\
8,4\end{array}$ & $\mid \begin{array}{rrrr}\text { Poids à la nais- } \\
\text { sance } & (\mathrm{kg}) & \ldots \\
\text { Poids à } & 30 & \text { jours } \\
\text { (kg) } & \ldots & \ldots \\
\text { Poids à } & 60 & \text { jours } \\
\text { (kg) } & \ldots \ldots \ldots \ldots \\
\text { Poids adulte } & (\mathrm{kg})\end{array}$ & $\begin{array}{l}0,450 \\
2,500 \\
5,000 \\
85-100\end{array}$ & $\begin{array}{c}1,5 \\
8-9 \\
18-20 \\
300-350\end{array}$ \\
\hline
\end{tabular}

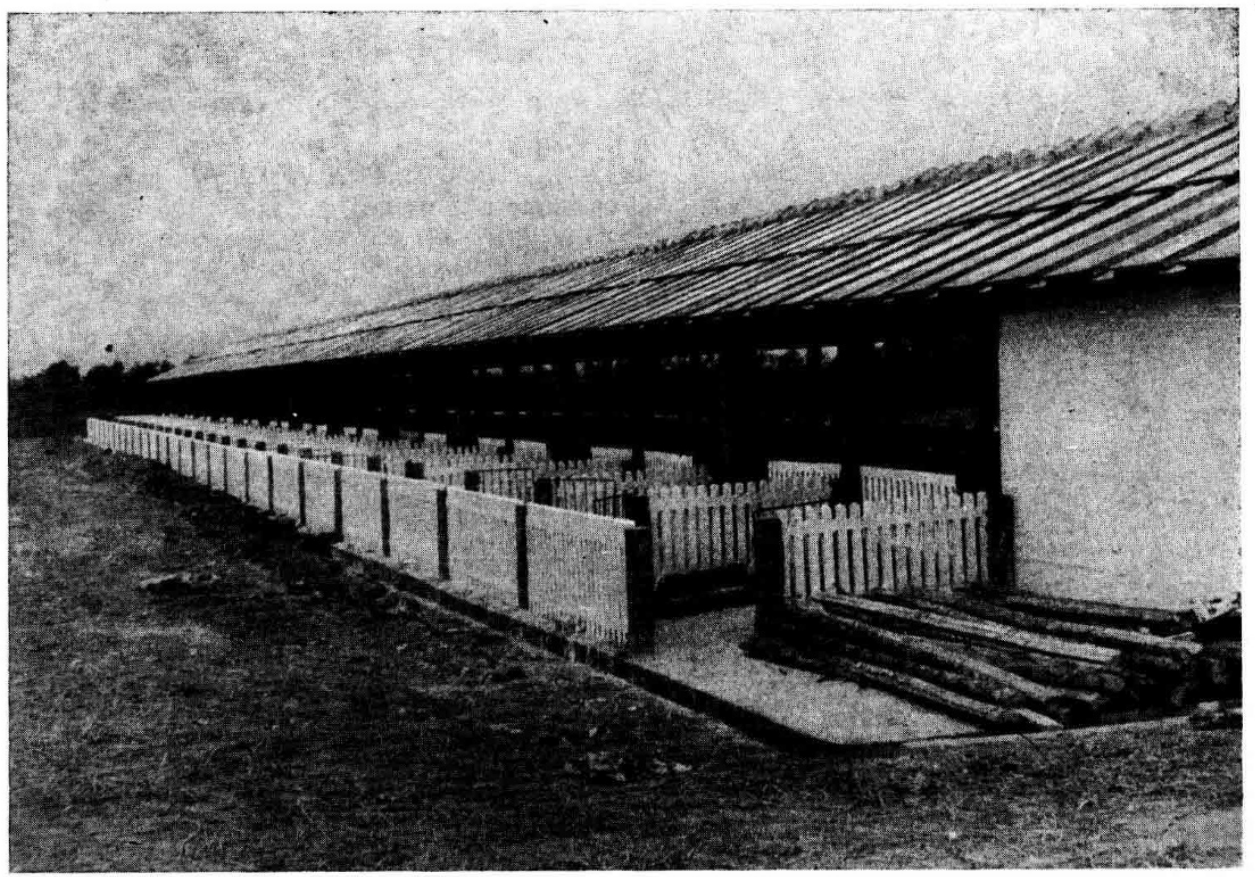

FIG. 5. - Porcherie d'une coopérative agricole 


\section{SUMMARY}

\section{THE VIETNAMESE $I^{\prime}$ BREED OF PIGS AND ITS CROSS WITH BERKSHIRE}

The $I^{\prime}$ breed is a small-sized pig, remarkable for its early reproduction, especially in males, and its prolificacy. Litter size at weaning, however, is limited by the low number of teats in the sow. The first cross with Berkshire improves growth and carcass quality : $22 \mathrm{~kg}$ more liveweight at ro months and 9 points more in percentage of meat in the carcass, relative to the purebred. Crossbred sows $\left(I^{\prime} \times\right.$ Berkshire $)$ show a reduced prolificacy but a $70 \mathrm{p}$. Ioo increase in birth-weight, relative to purebreds. Teats number in crossbreds is intermediate between the two parentil breeds. 